\title{
High Performance and Stable All-Inorganic Metal Halide Perovskite-Based Photodetectors for Optical Communication Applications
}

Chunxiong Bao, Jie Yang, Sai Bai, Weidong Xu, Zhibo Yan, Qingyu Xu, J unming Liu, Wenjing Zhang and Feng Gao

The self-archived postprint version of this journal article is available at Linköping University Institutional Repository (DiVA):

http:// urn.kb.se/ resolve?urn=urn:nbn:se:liu:diva-151638

N.B.: When citing this work, cite the original publication.

Bao, C., Yang, J., Bai, S., Xu, W., Yan, Z., Xu, Q., Liu, J ., Zhang, W., Gao, F., (2018), High Performance and Stable All-Inorganic Metal Halide Perovskite-Based Photodetectors for Optical Communication Applications, Advanced Materials, 30(38), 1803422. https:// doi.org/ 10.1002/ adma.201803422

Original publication available at:

https:/ / doi.org/ 10.1002/ adma.201803422

Copyright: Wiley (12 months)

http:// eu.wiley.com/WileyCDA/ 
High performance and stable all-inorganic metal halide perovskite-based photodetectors for optical communication applications

Chunxiong Bao, Jie Yang, Sai Bai, Weidong Xu, Zhibo Yan, Qingyu Xu, Junming Liu, Wenjing Zhang*, Feng Gao*

Dr. C. Bao, Prof. W. Zhang

International Collaborative Laboratory of 2D Materials for Optoelectronics Science and Technology\&Key Laboratory of Optoelectronic Devices and Systems of Ministry of Education and Guangdong Province, College of Optoelectronic Engineering, Shenzhen University, Shenzhen 518060, China

E-mail: wjzhang@szu.edu.cn.

Dr. C. Bao, Dr. J. Yang, Dr. S. Bai, Dr. W. Xu, Dr. Z. Yan, Prof. F. Gao

Department of Physics, Chemistry, and Biology (IFM), Linköping University, Linköping SE58183, Sweden

E-mail: feng.gao@liu.se

Dr. J. Yang, Prof. Q. Xu

School of Physics, Southeast University, Nanjing 211189, P. R. China

Dr. Z. Yan, Prof. J. Liu

Laboratory of Solid State Microstructures and Innovation Center of Advanced

Microstructures, Nanjing University, Nanjing 210093, P. R. China

Keywords: inorganic perovskite, photodetector, fast response, stability, optical communication 
Abstract: Photodetectors are critical parts of an optical communication system for achieving efficient photoelectronic conversion of signals and the response speed directly determines the bandwidth of the whole system. Metal halide perovskites, an emerging class of low-cost solution-processed semiconductors exhibiting strong optical absorption, low trap states and high carrier mobility, have been widely investigated in photodetection applications. In this work, through optimizing the device engineering and film quality, we demonstrate high-performance photodetectors based on all-inorganic cesium lead halide perovskite $\left(\mathrm{CsPbI}_{x} \mathrm{Br}_{3-\mathrm{x}}\right)$ which simultaneously possess high sensitivity and fast response. The optimized devices processed from $\mathrm{CsPbIBr}_{2}$ perovskite show a practically measured detectable limit of about $21.5 \mathrm{pW} \mathrm{cm}{ }^{-2}$ and a fast response time of 20 ns, which are both among the highest reported device performance of perovskite-based photodetectors. Moreover, the photodetectors exhibit outstanding long-term environmental stability, with negligible degradation of the photoresponse property after $2000 \mathrm{~h}$ under ambient conditions. In addition, for the first time, we successfully integrate the resulting perovskite photodetector into an optical communication system and demonstrate its applications as an optical signal receiver on transmitting the text and audio signals. Our results suggest that all-inorganic metal halide perovskite-based photodetectors have great application potentials for optical communication. 
Photodetectors are important for imaging and optical communication applications and are key components of many electronic products e.g. digital camera, smart phone, and medical diagnostics instrument. ${ }^{[1,2]}$ Most of the commercially available photodetectors are currently based on traditional crystalline inorganic semiconductors e.g. silicon (Si) and indium gallium arsenide (InGaAs), which need expensive high-vacuum manufacturing processes. Solutionprocessed semiconductors, such as organic semiconductors and quantum dots, have been widely studied as an alternative to inorganic semiconductors in photodetection, especially for some special applications, which require color tunability, large area or mechanical flexibility ${ }^{[3,4]}$ However, limited by their low carrier mobilities, most of the photodetectors based on organic semiconductors or quantum dots exhibit low response speed, hampering their practical applications in optical communication systems.

Solution-processed metal halide perovskites with large optical absorption coefficient and high carrier mobility, which have achieved great success in photovoltaic devices, have been intensively studied in the field of photodetection. ${ }^{[5-19]}$ However, up to now, rarely reported perovskite photodetector simultaneously show high sensitivity, fast response and good stability, which are among the most important characteristics of a photodetector for use in imaging and optical communication system. Previous reports have demonstrated that the device performance of photodetectors, especially the response speed, strongly depends on the device structure and the material properties of the active layer. ${ }^{[8,12,20,21]}$ Metal-semiconductor-metal (MSM) planar structure and p-i-n diode are the two widely used device architectures of perovskite photodetectors. Although the detection limit of perovskite photodetectors based on MSM structure have been significantly improved to sub-nW/ $\mathrm{cm}^{2},{ }^{223]}$ the absence of efficient charge blocking layer or built-in field in the device results in large dark noise current, limiting the further improvement of the detection limit. Meanwhile, the un-passivated defects in the perovskite film limit the response speed of MSM detectors with the reported fastest response in 
the range of tens of $\mu \mathrm{s} .{ }^{[24-26]}$ With significantly reduced dark current due to the use of efficient charge blocking layers, perovskite photodetectors based on p-i-n diode structure show much improved response sensitivity and response speed compare with their MSM counterparts. Previous reported perovskite photodetectors based on p-i-n device structure processed from either polycrystalline thin film or single crystal exhibit detectable limits of sub-pW/cm².[12,14] In addition, due to the existence of the built-in electric field and the efficient defects passivation by the charge selective layers, the response time of p-i-n perovskite photodetectors have been improved to sub-nanosecond, ${ }^{[16]}$ showing great potential in optical communication applications.

Long-term stability is important for perovskite photodetectors for practical applications. To improve the stability of perovskite photodetectors, all-inorganic cesium halide perovskites (AIHP) $\mathrm{CsPbX}_{3}(\mathrm{X}=\mathrm{Br}, \mathrm{I})$, which have been demonstrated with better materials stability, ${ }^{\text {[27-29] }}$ have been used to replace the hybrid perovskites in the photodetectors. ${ }^{[24-26,30-35]}$ However, most of the reported AIHP photodetectors are based on nano-, micro- or bulk crystals with a simple MSM structure and exhibit large dark current noise and low sensitivity. Meanwhile, the reported response speed ranges from tens $\mu$ s to hundreds ms, which hampers their application in imaging and optical communication.

Herein, we report high-performance photodetectors processed from all-inorganic $\mathrm{CsPbI}_{\mathrm{x}} \mathrm{Br}_{3-\mathrm{x}}$ perovskite thin films based on the p-i-n structure. We introduce polyethylenimine ethoxylated (PEIE) to modify the substrate and obtain $\mathrm{CsPbI}_{\mathrm{x}} \mathrm{Br}_{3-\mathrm{x}}$ film with much improved film quality. The resulting photodetectors based on $\mathrm{CsPbIBr}_{2}$ and $\mathrm{CsPbBr}_{3}$ show detectable limits of tens of pW $\mathrm{cm}^{-2}$ and fast response speed. Moreover, the devices based on AIHP show outstanding operational and environmental stability, with negligible degradation of the photoresponsivity after persistently operating during the test period ( $18 \mathrm{~h})$ and storage for $2000 \mathrm{~h}$ under ambient 
conditions. In addition, for the first time, we successfully demonstrate the use of our photodetectors in an optical communication system.

(a)
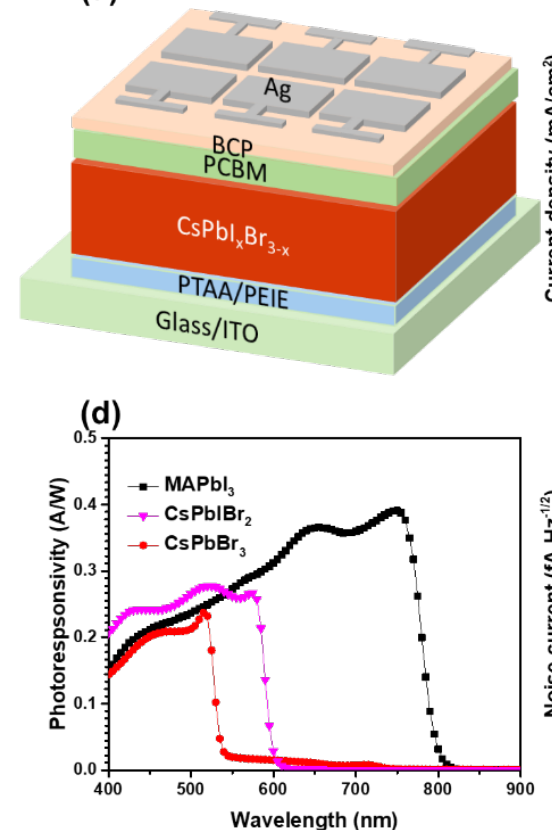

(b)

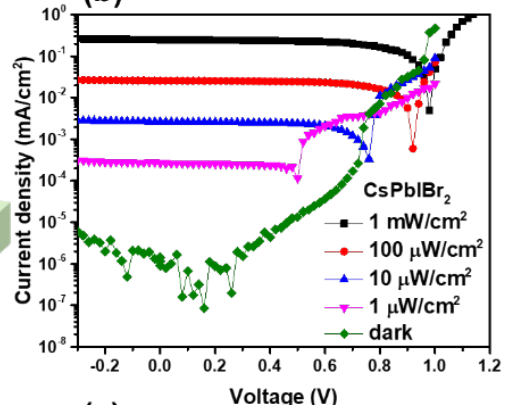

(e)

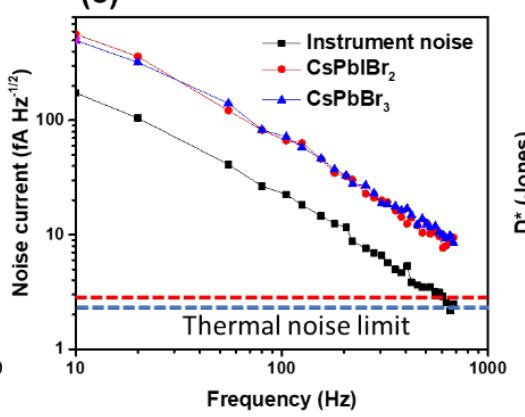

(c)

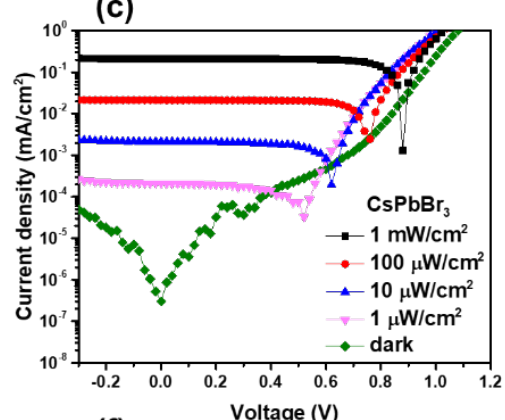

(f)

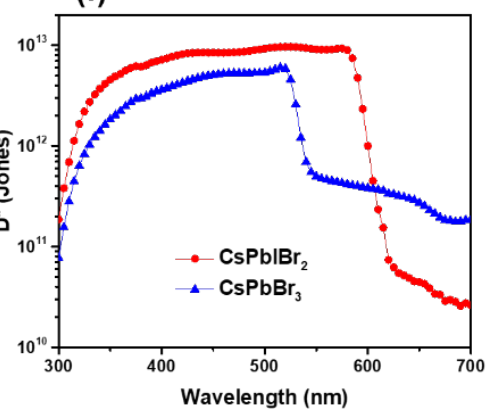

Figure 1. a) Schematic structure of photodetector based on all-inorganic perovskite $\mathrm{CsPb}_{\mathrm{x}} \mathrm{Br}_{3}$

x. b) and c) Current density-voltage (J-V) curves of $\mathrm{CsPbIBr}_{2}$ and $\mathrm{CsPbBr}_{3}$ based photodetectors under dark and illumination of $450 \mathrm{~nm}$ monochrome light with intensity of 1 $\mu \mathrm{m} \mathrm{cm} \mathrm{cm}^{-2}$ to $1 \mathrm{~mW} \mathrm{~cm}^{-2}$. d) Photoresponsivity spectra of photodetectors based on hybrid perovskite $\mathrm{CH}_{3} \mathrm{NH}_{3} \mathrm{PbI}_{3}\left(\mathrm{MAPbI}_{3}\right)$ and all-inorganic perovskite $\mathrm{CsPbI}_{\mathrm{x}} \mathrm{Br}_{3-\mathrm{x}}$. e) Noise current spectra of photodetectors based on $\mathrm{CsPbIBr}_{2}$ and $\mathrm{CsPbBr}_{3}$ as well as the instrument noise and thermal noise limit calculated based on the J-V curves under dark. f) Specific detectivity ( $D^{*}$ ) spectra of photodetectors based on $\mathrm{CsPbIBr}_{2}$ and $\mathrm{CsPbBr}_{3}$.

The schematic device structure of the AIHP photodetector is shown in Figure 1a. Here, p-type polymer poly[bis(4-phenyl) (2,4,6-trimethylphenyl)amine] (PTAA) is used as the holetransporting layer, small molecular [6,6]-phenyl-C61-butyric acid methyl ester (PCBM) and bathocuproine (BCP) as electron transport and exciton blocking layers, respectively. $\mathrm{CsPbI}_{\mathrm{x}} \mathrm{Br}_{3-\mathrm{x}}$ film is deposited via one-step spin-coating method. Although PTAA has been used as an efficient hole-transporting layer in previous reports using organic cation-contained perovskites, we notice the surface wettability problem when we deposit AIHP precursor on it 
(Figure S1 in the supporting information). To improve the coverage and quality of the AIHP film on PTAA, an amphiphilic PEIE is introduced on the surface of PTAA, making the film wetting to the precursor solution. As shown in Figure S1 and S2, the existence of PEIE significantly improves the film coverage of the $\mathrm{CsPbIBr}_{2}$ and $\mathrm{CsPbBr}$ films but little difference on the phase and crystallinity is observed. The cross-section scanning electron microscope (SEM) images of the devices shown in Figure S3 indicate the thicknesses of the $\mathrm{CsPbIr}_{2}$ and $\mathrm{CsPbBr}_{3}$ films used in our devices are about 300 and $120 \mathrm{~nm}$, respectively. The effect of the perovskite film thickness to the dark and photocurrent of the devices are studied by adjusting the precursor concentration. As shown in Figure S4, the dark current of the devices decrease with precursor concentration (from $3.6 \times 10^{-3}$ to $6.0 \times 10^{-6} \mathrm{~mA} \mathrm{~cm}^{-2}$ at $-0.3 \mathrm{~V}$ ) and the photocurrent of the devices increase with the precursor concentration (from 4.1 to $6.0 \mathrm{~mA} \mathrm{~cm}^{-2}$ at $0 \mathrm{~V}$ ) when the precursor concentration increase from $0.5 \mathrm{M}$ to $1.0 \mathrm{M}$, mainly because a thicker film leads to less pin-holes and better absorption of incident light with increasing thickness (Figure S5). The existence of the p- and n-type charge blocking layer and the high quality of the perovskite films significantly reduce the dark noise current of the obtained devices. As shown in Figure $\mathbf{1 b}$ and $\mathbf{c}$, the dark currents of the $\mathrm{CsPbIBr}_{2}$ and $\mathrm{CsPbBr}_{3}$ devices under $-0.3 \mathrm{~V}$ bias are around $6.0 \times 10^{-6} \mathrm{~mA} \mathrm{~cm}^{-2}$, and $4.8 \times 10^{-5} \mathrm{~mA} \mathrm{~cm}^{-2}$, respectively. The values are much lower than the MSM devices and comparable with previous reported high performance photodetectors based on organic-inorganic hybrid perovskite thin films and single crystals. ${ }^{[8,12,13,36]}$ A lower dark current indicates a lower shot noise and always along with a lower thermal noise, which is one of the key factors to achieve highly sensitive photodetectors. ${ }^{[12]}$ We show the current densityvoltage $(J-V)$ curves of the devices under illuminations of a $450 \mathrm{~nm}$ monochrome light with different intensities in Figure 1b and c. We measure the photocurrents of the devices of about $2 \times 10^{-4} \mathrm{~mA} \mathrm{~cm}^{-2}$ under low light intensity $\left(1 \mu \mathrm{W} \mathrm{cm} \mathrm{cm}^{-2}\right)$. We observe excellent linear response property of our devices to the light intensity when we increase the light intensity from 1 to 10 , $100 \mu \mathrm{W} \mathrm{cm}{ }^{-2}$ and finally to $1 \mathrm{~mW} \mathrm{~cm}^{-2}$ by order of magnitude. $\left(\mathrm{J} \propto \mathrm{P}^{\mathrm{n}}\right.$, if $\mathrm{n}=1$, it’s linear 
dependence of photocurrent on incident light power density, indicating high separation efficiency of photogenerated carriers)

The photoresponsivity and the dark current noise are two key factors that determine the device sensitivity. We show the photoresponsivity spectra of devices based on AIHP and compare it with devices based on methylammonium lead iodide perovskite $\left(\mathrm{MAPbI}_{3}\right)$ in Figure 1d. The $\mathrm{CsPbIBr}_{2}$ device shows responsivities from 0.21 to $0.28 \mathrm{~A} \mathrm{~W}^{-1}$ at the wavelength range from 400 to $580 \mathrm{~nm}$, which is comparable with that of $\mathrm{MAPbI}_{3}$ device in the same wavelength range (Figure 1d). The responsivities of the $\mathrm{CsPBr}_{3}$ device are 0.08 to $0.13 \mathrm{~A} \mathrm{~W}^{-1}$ at the wavelength range of 400 to $520 \mathrm{~nm}$. The photoresponsivity spectra are corresponding to the absorption spectra of the perovskite films (Figure S6) indicating the response are from the perovskite films. Before measuring the device noise with a lock-in amplifier, the noise floor of the instrument is determined. As shown in Figure 1e, the noise of the instrument is 180 to $2.3 \mathrm{fA} \mathrm{Hz}^{-1 / 2}$ from 10 to $700 \mathrm{~Hz}$. The measured noise currents of the $\mathrm{CsPbIBr}_{2}$ and $\mathrm{CsPbBr}_{3}$ devices are 567 to 7.7 fA $\mathrm{Hz}^{-1 / 2}$, and 500 to $8.5 \mathrm{fA} \mathrm{Hz}^{-1 / 2}$, respectively, which are obviously larger than the instrument noise floor, indicating the measured noise is mainly from the devices. Figure 1e also shows the thermal noise limit of the devices calculated from the dark deferential resistance based on the dark $J$ - $V$ curves shown in Figure 1b and c. The measured noises of the devices are larger than the thermal noise limit at the measurement frequency range, which indicates that the measured noise is dominated by $1 / \mathrm{f}$ noise. Based on the measured noise and responsivity, the noise equivalent power (NEP) of the $\mathrm{CsPbIBr}_{2}$ and $\mathrm{CsPbBr}_{3}$ devices at high frequency $(>700 \mathrm{~Hz})$ can be calculated to be smaller than $2.7 \times 10^{-14}$ and $3.5 \times 10^{-14} \mathrm{~W} \mathrm{~Hz}^{-1 / 2}$, respectively, which are comparable to the reported best performing perovskite photodetectors (see Table 1). Along with the active area of the device, the specific detectivity $\left(D^{*}\right)$, another figure of merit of the photodetector which is related to the sensitivity that a detector can distinguish from the background noise can be calculated based on the responsivity and noise. Figure 1f shows the 
$D^{*}$ spectra of $\mathrm{CsPbIBr}_{2}$ and $\mathrm{CsPbBr}_{3}$ devices. The peak $D^{*}$ of about $9.7 \times 10^{12}$ and $6.0 \times 10^{12} \mathrm{~cm}$ $\mathrm{W}^{-1} \mathrm{~Hz}^{1 / 2}$ are obtained at around $525 \mathrm{~nm}$ and $515 \mathrm{~nm}$ for $\mathrm{CsPbIBr}_{2}$ and $\mathrm{CsPbBr}_{3}$ devices, respectively, which are among the highest detectivity of the reported perovskite photodetectors (Table 1).

(a)

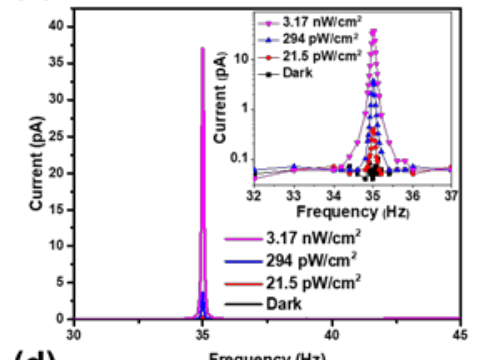

(d)

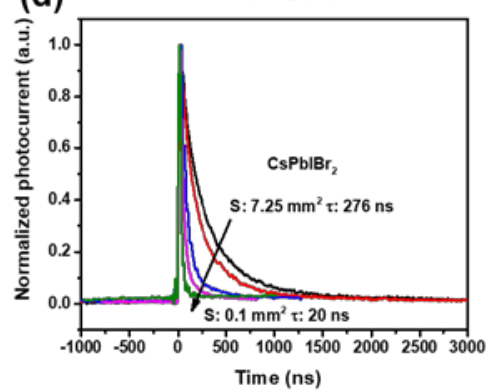

(b)

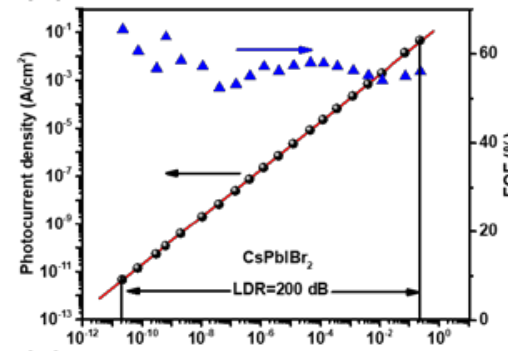

(e)

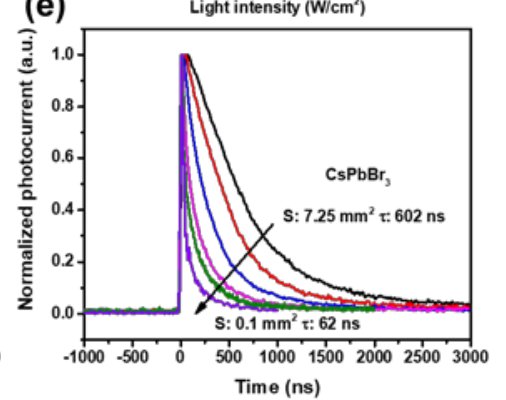

(c)

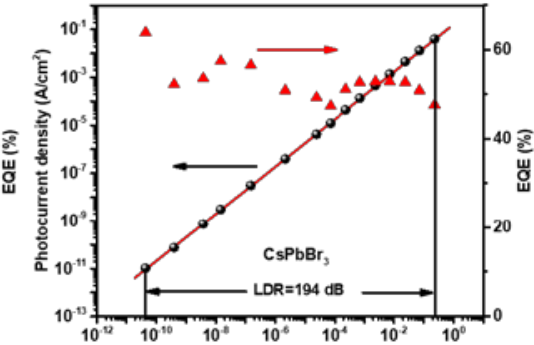

(f)

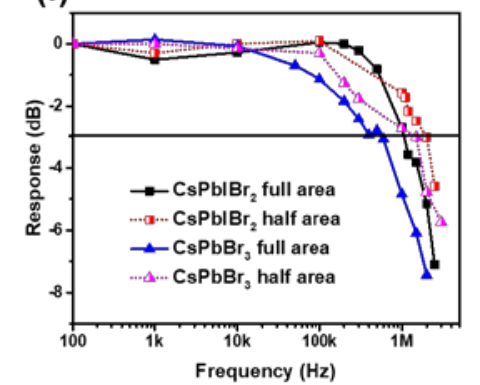

Figure 2. a) Current spectra of $\mathrm{CsPbIBr}_{2}$ photodetector under dark and week light. b) and c) Photocurrent and EQE curves of $\mathrm{CsPbIBr}_{2}$ and $\mathrm{CsPbr}_{3}$ photodetectors under different light intensities. The linear dynamic range (LDR) of $\mathrm{CsPbIBr}_{2}$ and $\mathrm{CsPbBr}_{3}$ photodetectors determined from the curve are 200 and 194 dB. d) and e) Transient photocurrent curves of $\mathrm{CsPbIBr} 2$ and $\mathrm{CsPbBr}_{3}$ based photodetectors with different active areas. f) frequency response of $\mathrm{CsPbIBr}_{2}$ and $\mathrm{CsPbBr}_{3}$ based photodetectors.

Photoresponse of the photodetectors under weak light is studied using the lock-in amplifier. A $450 \mathrm{~nm}$ LED modulated with a $35 \mathrm{~Hz}$ square wave is used as the light source. Neutral density filters and a Si photodetector are used to adjust and calibrate the light intensities. Figure 2a shows the photocurrent spectra of the $\mathrm{CsPbIBr}_{2}$ photodetector under dark and weak light intensities from $21.5 \mathrm{pW} \mathrm{cm}^{-2}$ to $3.17 \mathrm{nW} \mathrm{cm}^{-2}$. The currents of the device under dark at different frequencies are about tens of fA, which is close to the noise current of the devices shown in Figure 1e. Sharp peaks at $35 \mathrm{~Hz}$, which is the modulation frequency of the incident light, can 
be found under light, indicating the current peaks are the signals of photodetector responding to the incident light. When the incident light intensity is about $21.5 \mathrm{pW} \mathrm{cm}{ }^{-2}$, an obvious peak at $35 \mathrm{~Hz}$ can be distinguished from the noise floor (inset of Figure 2a), indicating the photodetector can detect the weak light as low as $21.5 \mathrm{pW} \mathrm{cm}^{-2}$. The current at $35 \mathrm{~Hz}$ increases linearly with the light intensities when it increases from $21.5 \mathrm{pW} \mathrm{cm}^{-2}$ to $3.17 \mathrm{nW} \mathrm{cm}^{-2}$.

Table 1. Summary the performance of photodetectors based on hybrid perovskite and allinorganic perovskite.

\begin{tabular}{|c|c|c|c|c|c|c|}
\hline Structure ${ }^{\text {a) }}$ & $\begin{array}{l}\mathrm{NEP}\left[\mathrm{W} \mathrm{Hz}^{-}\right. \\
\end{array}$ & $\begin{array}{l}\text { Drive } \\
\text { Voltage } \\
{[\mathrm{V}]}\end{array}$ & $\begin{array}{l}\mathrm{D}^{*}\left[\begin{array}{ll}\mathrm{cm} & \mathrm{W}^{-1} \\
\left.\mathrm{~Hz}^{1 / 2}\right]\end{array}\right.\end{array}$ & $\begin{array}{l}\text { Rising/falling } \\
\text { time (active area) }\end{array}$ & Stability & Ref \\
\hline ITO/MAPbI ${ }_{3} / \mathrm{ITO}$ & & 3 & & $\sim 0.1 \mathrm{~s}$ & & {$[5]$} \\
\hline $\mathrm{Au} / \mathrm{MAPbI}_{3} / \mathrm{Au}$ & & 10 & $1.0 \times 10^{12}$ & $0.3 \mathrm{~ms} / 0.4 \mathrm{~ms}$ & $\sim 4$ weeks & [7] \\
\hline PEDOT:PSS/MAPbI $I_{3-\mathrm{x}} \mathrm{Cl}_{x} / \mathrm{PCBM}$ & $4.2 \times 10^{-12}$ & 0 & & $160 \mathrm{~ns}$ & & [8] \\
\hline OTPD/MAPbI ${ }_{3} / \mathrm{PCBM} / \mathrm{C}_{60}$ & $4.3 \times 10^{-14}$ & 0 & $7.4 \times 10^{12}$ & $120 \mathrm{~ns}$ & & {$[12]$} \\
\hline $\mathrm{PTAA} / \mathrm{MAPbI}_{3} / \mathrm{C}_{60} / \mathrm{BCP}$ & $3.8 \times 10^{-11}$ & 0 & $7.8 \times 10^{12}$ & $\begin{array}{l}0.95 \mathrm{~ns} \\
\left(0.01 \mathrm{~mm}^{2}\right)\end{array}$ & & {$[21]$} \\
\hline $\mathrm{PTAA} / \mathrm{MAPbBr}_{3} \mathrm{SC} / \mathrm{C}_{60} / \mathrm{BCP}$ & $5.4 \times 10^{-15}$ & 0 & $1.5 \times 10^{13}$ & $100 \mathrm{~ns}$ & & [14] \\
\hline $\mathrm{Au} / \mathrm{CsPbBr}{ }_{3} \mathrm{NW} / \mathrm{Au}$ & & 3 & & $252 \mu \mathrm{s}$ & & {$[25]$} \\
\hline $\mathrm{Au} / \mathrm{CsPbBr}{ }_{3} / \mathrm{Au}$ & & 6 & & $430 \mu \mathrm{s} / 318 \mu \mathrm{s}$ & & {$[31]$} \\
\hline $\mathrm{Au} / \mathrm{CsPbBr}{ }_{3} \mathrm{NS}-\mathrm{CNT} / \mathrm{Au}$ & & 10 & & $16 \mu \mathrm{s} / 380 \mu \mathrm{s}$ & & {$[23]$} \\
\hline $\mathrm{Au} / \mathrm{CsPbBr}_{3} \mathrm{NP} / \mathrm{Au}$ & & 1.5 & $7.5 \times 10^{12}$ & $600 \mu \mathrm{s} / 900 \mu \mathrm{s}$ & & [32] \\
\hline $\mathrm{Au} / \mathrm{CsPbBr}{ }_{3} \mathrm{SC} / \mathrm{Au}$ & & 10 & & $69 \mu \mathrm{s} / 261 \mu \mathrm{s}$ & & {$[35]$} \\
\hline $\mathrm{Au} / \mathrm{CsPbBr}{ }_{3} \mathrm{SC} / \mathrm{Au}$ & & 30 & & $0.3 \mathrm{~s} / 0.3 \mathrm{~s}$ & & {$[34]$} \\
\hline $\mathrm{Au} / \mathrm{CsPbBr}_{3} \mathrm{QD} / \mathrm{Au}$ & & 9 & & $1.8 \mathrm{~ms} / 1.0 \mathrm{~ms}$ & $>2$ months & {$[30]$} \\
\hline ITO/CsPbBr ${ }_{3} \mathrm{MC} / \mathrm{ITO}$ & & 3 & $\sim 10^{13}$ & $0.5 \mathrm{~ms} / 1.6 \mathrm{~ms}$ & & [33] \\
\hline PTAA/PEIE/CsPbIBr 2 /PCBM/BCP & $2.7 \times 10^{-14}$ & 0 & $9.7 \times 10^{12}$ & $20 \mathrm{~ns}\left(0.1 \mathrm{~mm}^{2}\right)$ & $>2000 \mathrm{~h}$ & This work \\
\hline $\mathrm{PTAA} / \mathrm{PEIE} / \mathrm{CsPbBr} \mathrm{P}_{3} / \mathrm{PCBM} / \mathrm{BCP}$ & $3.5 \times 10^{-14}$ & 0 & $6.0 \times 10^{12}$ & $62 \mathrm{~ns}\left(0.1 \mathrm{~mm}^{2}\right)$ & $>2000 \mathrm{~h}$ & This work \\
\hline
\end{tabular}

a) SC: single crystal, NW: nanowire, NS: nanosheet, CNT: carbon nanotube, NP: nanoplate, QD: quantum dot, MC: microcrystal

The linear response of a photodetector to the light intensity is a key figure of merit to a photodetector, especially for use in light measurement and imaging applications. As we show in Figure 2b and 2c, to determine the linear response range of the photodetector, we adjust the incident light intensities from $\mathrm{pW} \mathrm{cm}^{-2}$ to hundreds $\mathrm{mW} \mathrm{cm}{ }^{-2}$ and measure the photocurrent of 
the photodetector. The photocurrent of the device based on $\mathrm{CsPbIBr}_{2}$ increases linearly from 4.6 $\times 10^{-12}$ to $4.7 \times 10^{-2} \mathrm{~A} \mathrm{~cm}^{-2}$ when the incident light intensities increase from $2.1 \times 10^{-11}$ to 0.23 $\mathrm{W} \mathrm{cm}{ }^{-2}$. The results indicate that the device can keep linear response to the light intensity over about 10 orders of magnitude, corresponding to a linear dynamic range (LDR) of about $200 \mathrm{~dB}$, which is close to that of previous reported perovskite photodetectors. ${ }^{[6,8,12]}$ The EQE of the photodetector is nearly constant $(57.1 \pm 3.1 \%)$ within this light intensity range, which confirms the excellent linear response of the device. As shown in Figure 2c, photodetector based on $\mathrm{CsPbBr} 3$ also shows excellent linear response and keeps a nearly constant EQE of $52.8 \pm 4.0 \%$ from $\mathrm{pW} \mathrm{cm}^{-2}$ to hundreds $\mathrm{mW} \mathrm{cm}^{-2}$, corresponding a broad LDR of $194 \mathrm{~dB}$.

We characterize the response speed of the AIHP photodetector at 0 bias voltage with a pulsed laser and an oscilloscope. Figure 2d and e show the transient photocurrent (TPC) curves of the $\mathrm{CsPbIBr}_{2}$ and $\mathrm{CsPbBr}_{3}$ devices with different active areas from $7.25 \mathrm{~mm}^{2}$ to around $0.1 \mathrm{~mm}^{2}$. By fitting the TPC curves with a single exponential function, the response time of the CsPbIBr 2 and $\mathrm{CsPBrr}_{3}$ devices with active areas of $7.25 \mathrm{~mm}^{2}$ are determined to 276 and $602 \mathrm{~ns}$, respectively. We observe that the response time obviously decrease with increasing active area, with the values decreasing to 20 and 62 ns for $\mathrm{CsPbIBr}_{2}$ and $\mathrm{CsPbBr}_{3}$ devices when the active area is $0.1 \mathrm{~mm}^{2}$, respectively, indicating the response time is dominated by the resistancecapacitance (RC) constant. ${ }^{[21,22]}$ As the input resistance is constant (50 $\Omega$ ), the RC constant is determined by the capacitance of the device which decreases with decreasing active area, so the response time decrease with decreasing active area. The response of our devices to the modulated light at different frequencies are shown in Figure $\mathbf{2 f}$ (the response waveforms of the devices can be seen in Figure S7). The 3dB cut-off frequency of $\mathrm{CsPbIr}_{2}$ and $\mathrm{CsPbBr}_{3}$ devices with full area of $7.25 \mathrm{~mm}^{2}$ are 0.6 and $1.0 \mathrm{MHz}$, respectively. When the active areas are reduced to a half, the $3 \mathrm{~dB}$ cut-off frequency increase to 1.5 and $3.0 \mathrm{MHz}$, respectively. The response 
speed of our AIHP photodetectors surpasses all the reported AIHP crystal photodetectors and is comparable to the reported fastest hybrid perovskite photodetectors (Table 1).

(a)

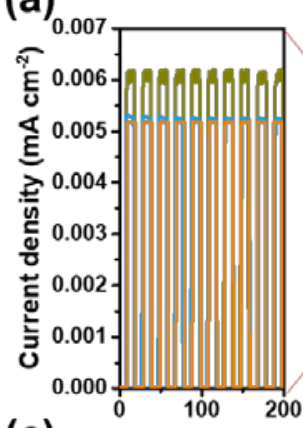

(c) Time (s)

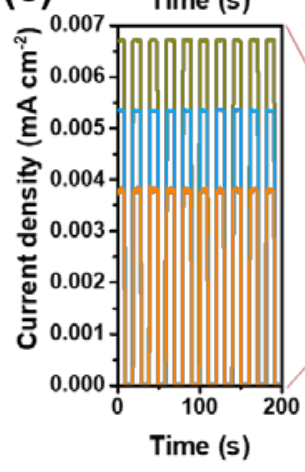

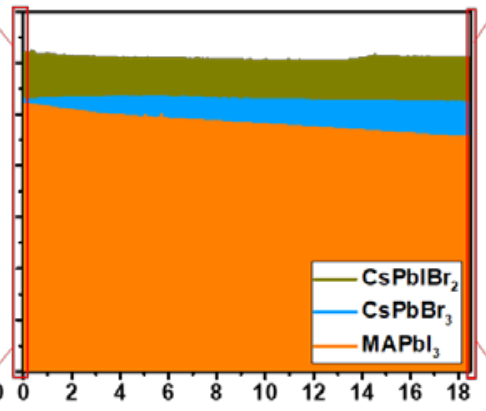

Time (h)

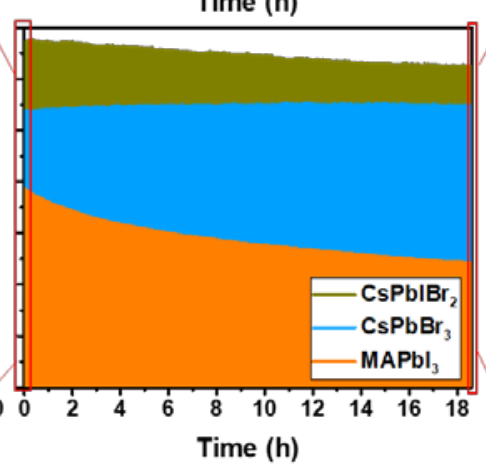

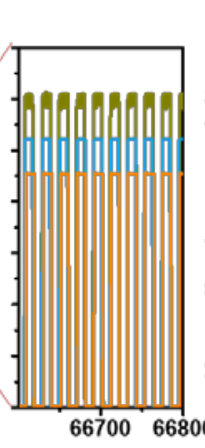

Time (s)

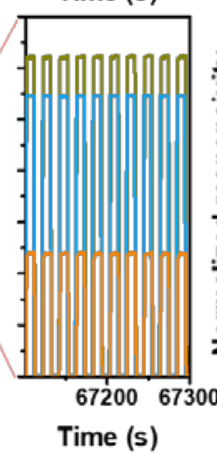

(b)

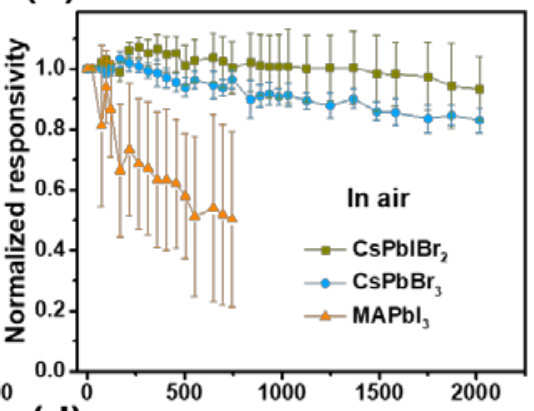

(d) Time (h)

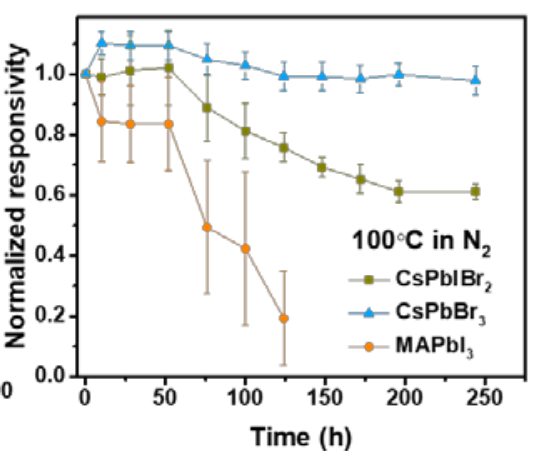

Figure 3. a) The response of fresh photodetectors based on $\mathrm{CsPbIBr}_{2}, \mathrm{CsPbBr}_{3}$ and $\mathrm{MAPbI}_{3}$ (at 0 bias voltage) to a $450 \mathrm{~nm}$ on/off modulated LED light $\left(\sim 20 \mu \mathrm{W} \mathrm{cm}{ }^{-2}\right)$ in a test period of about 18 hours. The left and right panels show the enlarged detail of the response at the first and last 200 seconds, respectively. b) Photoresponsivity evolution of photodetectors based on inorganic perovskite $\mathrm{CsPbI}_{\mathrm{x}} \mathrm{Br}_{3-\mathrm{x}}$ and hybrid perovskite $\mathrm{MAPbI}_{3}$ in air ambition condition without encapsulation. c) The response of photodetectors based on $\mathrm{CsPbIBr}_{2}, \mathrm{CsPbBr}_{3}$ and $\mathrm{MAPbI}_{3}$ after stored in air for 1000 hours in a test period of about 18 hours. The left and right panels show the enlarged detail of the response at the first and last 200 seconds, respectively. d) Photoresponsivity evolution of photodetectors based on inorganic perovskite $\mathrm{CsPbI}_{\mathrm{x}} \mathrm{Br} 3-\mathrm{x}$ and hybrid perovskite $\mathrm{MAPbI}_{3}$ at $100{ }^{\circ} \mathrm{C}$ in $\mathrm{N}_{2}$ ambient condition.

We investigate the reliability of the un-encapsulated AIHP photodetectors under working conditions and their long-term stability in ambient. Figure 3a shows response of the fresh AIHP devices under illumination of a $450 \mathrm{~nm}$ blue light with intensity of $\sim 20 \mu \mathrm{W} \mathrm{cm}{ }^{-2}$. The dark and photo current of the $\mathrm{CsPbIBr}_{2}$ and $\mathrm{CsPbBr}_{3}$ devices show little difference throughout the whole test period of more than $18 \mathrm{~h}$, while the $\mathrm{MAPbI}_{3}$ device shows a $12 \%$ attenuation. This is not 
surprising since the AIHP materials have been demonstrated with superior stability compared with the hybrid ones, especially under illumination in air, which the $\mathrm{MAPbI}_{3}$ experience fast degradation due to the accelerated ion migration and possibly formed superoxide components. ${ }^{[37-39]}$ We further show the long-term ambient stability of the photoresponsivity of the AIHP photodetectors and $\mathrm{MAPbI}_{3}$ devices in Figure 3b. We observe that the photoresponsivity of $\mathrm{MAPbI}_{3}$ device decrease to about $50 \%$ of its initial value at the first stage of about 700 hours. In contrast, the AIHP based photodetectors show much better ambient stability under the same aging conditions, with only 7 and $13 \%$ degradation on the device responsibility are observed after more than $2000 \mathrm{~h}$. We repeat the test on the device reliability after $1000 \mathrm{~h}$ aging under ambient and show the results in Figure 3c. We observe that $\mathrm{CsPbIBr} 2$ and $\mathrm{CsPbBr}_{3}$ devices show stable and repeatable photocurrent as the fresh devices. About 93\% and almost $100 \%$ photocurrent can still be maintained at the end of the test period (more than $18 \mathrm{~h}$ ) for $\mathrm{CsPbIBr}_{2}$ and $\mathrm{CsPbBr} 3$ photodetectors, while only $61 \%$ of the initial photocurrent can be maintained for $\mathrm{MAPbI}_{3}$ device after this test period.

We also carry out the thermal stability test of the AIHP and $\mathrm{MAPbI}_{3}$ photodetectors under $100^{\circ} \mathrm{C}$ in a nitrogen filled glovebox, as shown in Figure 3d. We find that the photoresponsivity of $\mathrm{MAPbI}_{3}$ devices experienced a fast decay to $80 \%$ of its initial value within $24 \mathrm{~h}$, and then degrade quickly to almost zero within $125 \mathrm{~h}$. The fast degradation of the $\mathrm{MAPbI}_{3}$ photodetectors under heating can be attributed to the thermal instable nature of the active material (Figure S8), which has been intensively reported. ${ }^{[40,41]}$ In contrast, we observe excellent thermal stability of the $\mathrm{CsPbIBr}_{2}$ and $\mathrm{CsPbBr}_{3}$ devices during the test. After about 244 hours heating, the photoresponsivity of $\mathrm{CsPbBr}_{3}$ devices does not show any obvious degradation, and that of $\mathrm{CsPbIBr}_{2}$ devices degrade about $40 \%$, which can be contributed to contact corrosion due to the reaction between $\mathrm{I}$ in the perovskite active layer and the $\mathrm{Ag}$ 
electrodes under high temperature. ${ }^{[42]}$ The stability results of our AIHP photodetectors represent the best operational stability and environmental stability so far (Table 1). ${ }^{[7,30]}$

(a)

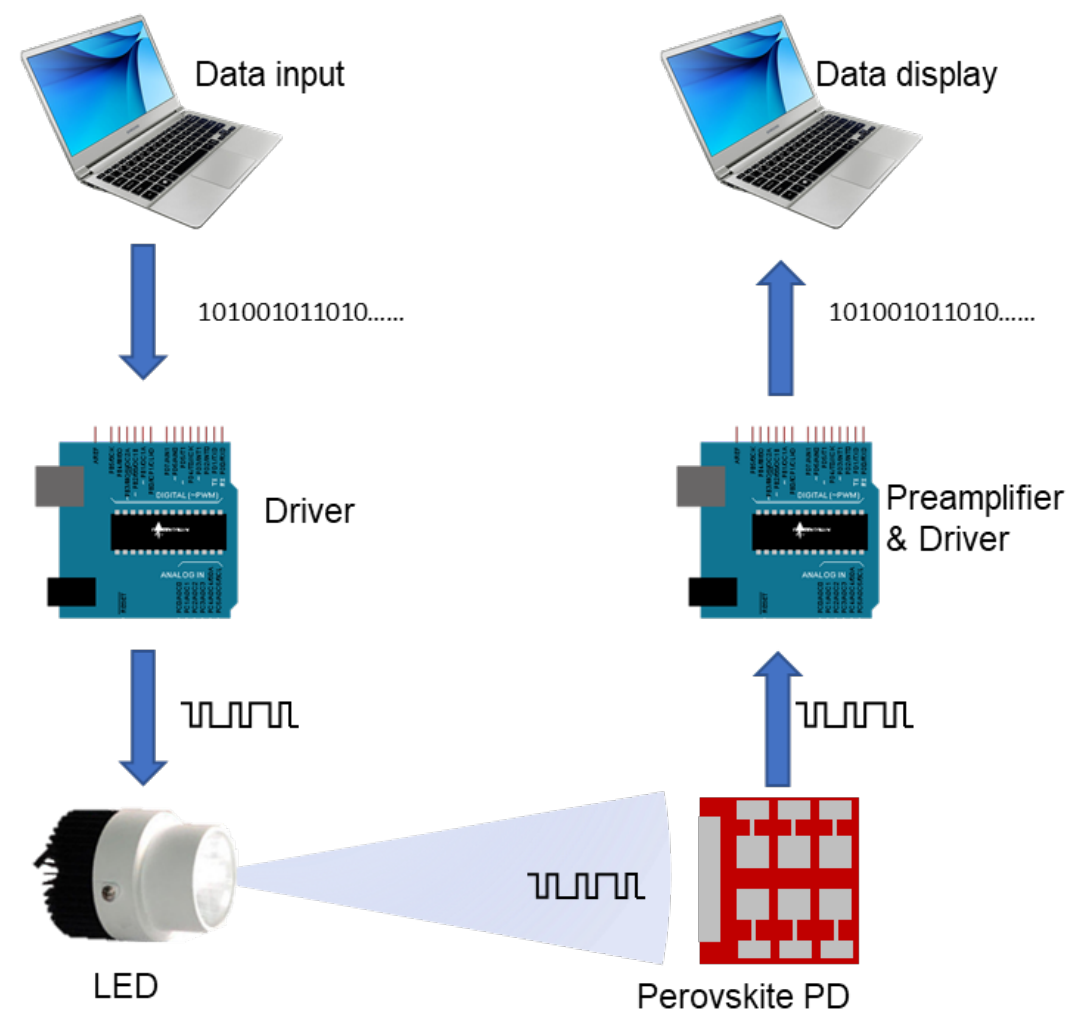

(b)

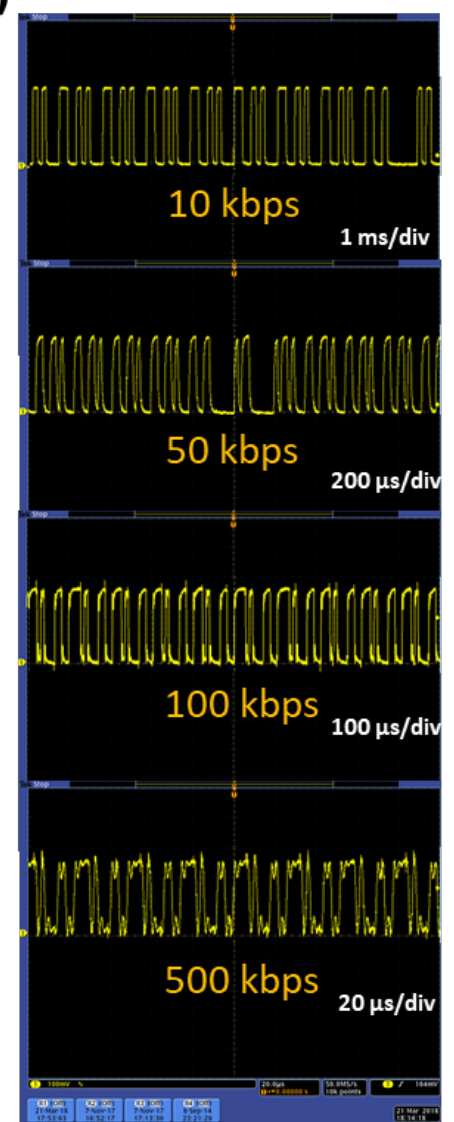

Figure 4. a) Schematic diagram of the visible light communication based on inorganic perovskite photodetector. b) The waveforms of the digital data received by the perovskite photodetector at different transmitted rate.

We further integrate the AIHP perovskite photodetectors into a visible light communications (VLC) system as a light signal receiver. VLC is a visible light based wireless communication technique emerging in recent years, featuring high speed, high security, low-cost energy and electromagnetic interference immunity. ${ }^{[43,44]}$ Unlike fiber-optic communication, which focus light from a LED or laser diode into a thin fiber, VLC is based on light divergently propagate in free space. In order to get enough signal to noise ratio $(\mathrm{S} / \mathrm{N})$ in VLC, more sensitive and larger area photodetectors are needed. Here, a prototype VLC system integrated with our AIHP photodetector as receiver is built up. As shown in Figure 4a, data flow from a computer were 
transferred into high and low voltage level using a driver. The voltage level was used to drive a LED to produce a modulated light, which can be received by an AIHP photodetector and produce high- and low-level photocurrent. The photocurrent is firstly amplified with a low noise current to voltage preamplifier and then input to a driver, which transfers the voltage signal into data and collected by computer. Figure $\mathbf{4 b}$ shows the current signal from the AIHP photodetector which receives the massage-modulated light at different bit rate, indicating the system can transmit data with a bit rate of $500 \mathrm{kbps}$. Based on the fast response speed of our AIHP photodetector showed in Figure 2, we anticipate that it should be possible to work at bit rate larger than $1 \mathrm{Mbps}$, but the practical rate is limited by the bandwidth of the preamplifier. The digital photograph of the VLC system is shown in Figure S9 in SI. The demonstration of the text and audio transmission via the VLC system can be seen in the video in SI.

In summary, we demonstrated high performance p-i-n structured photodetectors based on all inorganic halide perovskite $\mathrm{CsPbI}_{\mathrm{x}} \mathrm{Br}_{3-\mathrm{x}}$. The obtained photodetectors show high sensitivity, and fast response speed and excellent linear dynamic range closed to $200 \mathrm{~dB}$. Meanwhile, the photodetectors show outstanding light stability, air stability and thermal stability compared to devices based on organic-inorganic hybrid perovskite. We successfully integrated the $\mathrm{CsPbIBr} 2$ photodetector into the visible light communication system and demonstrated the first perovksite detector-based prototype signal receiver. Our results suggest that high speed and stable perovskite detectors may have great potential for optical communication applications.

\section{Experimental Section}

Device fabrication: Patterned ITO glass substrates were cleaned by heating in ammonia and hydroperoxide blend solution in deionized water at $80{ }^{\circ} \mathrm{C}$ for 30 minutes and rinsed with deionized water. The cleaned substrates were dried in nitrogen flow and treated with UV-ozone for 20 minutes before used. PTAA ( $2 \mathrm{mg} \mathrm{mL}^{-1}$ in toluene) and PEIE (0.05 wt\% in isopropanol) 
solution were successively spin coated on the substrates at $4000 \mathrm{rpm}$ and $5000 \mathrm{rpm}$ for $30 \mathrm{~s}$ and heated at $100{ }^{\circ} \mathrm{C}$ for 10 minutes for each layer. Precursor solution of $\mathrm{CsPbIBr}_{2}(1.0 \mathrm{M}$ in DMSO) and $\mathrm{CsPbBr}_{3}(0.5 \mathrm{M}$ in DMSO) were spin coated on the PTAA/PEIE layer at $4000 \mathrm{rpm}$ for 2 minutes and 1 minute, respectively. Then the films were annealed on a hot plate at $100{ }^{\circ} \mathrm{C}$ for 10 minutes. After cooling down to the room temperature, the perovskite films were spin coated with PCBM and BCP layers successively as electron transfer and exciton blocking layers using PCBM (20 mg mL $\left.\mathrm{m}^{-1}\right)$ solution in chlorobenzene and BCP (2 mg mL $\left.\mathrm{mL}^{-1}\right)$ solution in isopropanol at $4000 \mathrm{rpm}$ for $30 \mathrm{~s}$. Finally, silver electrodes ( 100 nm in thickness) were thermally deposited with a mask to define the active area as $7.25 \mathrm{~mm}^{2}$. For all the spin coating processes, the substrates start to spin after loading the solution, and the rotation was speed up with an accelerated speed of $2000 \mathrm{rpm} / \mathrm{s}$.

Device characterization: Current density-voltage $(\mathrm{J}-\mathrm{V})$ curves of the devices were measured using a Keithley 2400 source meter. The monochrome light was from a LED with center peak at $450 \mathrm{~nm}$. The light intensity was tuned by changing the current through the LED and calibrated using a Hamamatsu Si photodiode (S1133). External quantum efficiency (EQE) of the devices were measured using a solar cell spectral response measurement system (QE-R3011, Enli Technology Co. Ltd). Before measurement, the system was calibrated using a standard Si crystalline solar cell. The responsivity $\left(R_{\lambda}\right)$ of the devices can be obtained via the following relationship:

$$
R_{\lambda}=\frac{\lambda \cdot E Q E}{h c / e}=\frac{\lambda \cdot E Q E}{1.24 \times 10^{-6}}(\mathrm{~A} / \mathrm{W})
$$

where $\lambda$ is the wavelength, $h$ the Plank constant, $c$ the light speed, $e$ the elementary charge. The noise current of the devices was measured using a lock-in amplifier (Stanford Research System, SR830). During the measurement, a grounded metal box was used to keep the device in dark and reduce the noise produced by the electromagnetic interference. The thermal noises of the devices are calculated based on the following equation: 


$$
i_{n, t}=\sqrt{\frac{4 k_{B} T B}{R}}
$$

where $k_{B}$ is the Boltzmann constant, $T$ the temperature, $B$ the bandwidth, and $R$ the differential resistance at $0 \mathrm{~V}$ extracted from the dark current shown in Figure $1 \mathrm{~b}$ and c. With the measured photoresponsivity and dark noise current $\left(i_{n}\right)$, the noise equivalent power $(N E P)$ and specific detectivity $\left(D^{*}\right)$ can been obtain using the following equation:

$$
\begin{aligned}
& N E P=\frac{i_{n}}{R_{\lambda}} \\
& D^{*}=\frac{R_{\lambda} \sqrt{A}}{i_{n}}
\end{aligned}
$$

here, the $i_{n}$ is chosen as the value measured at $700 \mathrm{~Hz}, R_{\lambda}$ the peek value of the measured photoresponsivity spectrum, A the active area of the devices. To measure the photocurrent of the devices under different light intensities, the LED was modulated with a square wave voltage generated from a function generator, and the light intensity was adjusted with neutral density filters and calibrated with the Si photodiode. The photocurrents of the devices were firstly amplified using a low noise preamplifier (Stanford Research System, SR570) and then measured using the lock-in amplifier. The linear dynamic range is calculated using the following relation:

$$
L D R=20 \log \frac{P_{\text {high }}}{P_{\text {low }}}=20 \log \frac{J_{\text {high }}}{J_{\text {low }}}
$$

where $P_{\text {high }}\left(J_{\text {high }}\right)$ and $P_{\text {low }}\left(J_{\text {low }}\right)$ are the highest and the lowest light intensity (current density) among which the device can keep linear response to the light intensity. Transient photocurrent of the devices was recorded by a digital oscilloscope (Tektronix, DPO 4104B-1) with an input resistance of $50 \Omega$, when the device was illuminated with a pulse laser whose wavelength is $337 \mathrm{~nm}$ and pulse width is $4 \mathrm{~ns}$ from a nitrogen laser (Stanford Research System, NL100). To measure the frequency response of the devices, a sinusoidal wave voltage driven LED was used as light source, and the photocurrents of the devices were recorded by the oscilloscope. When measuring the ambient stability, the devices without encapsulation were stored under ambient 
condition and dark and their photoresponsivities of the devices in some specific time were measured using the lock-in amplifier and the modulated blue LED. When measuring the thermal stability, devices without encapsulation were heated in a hot plate at $100{ }^{\circ} \mathrm{C}$ in $\mathrm{N}_{2}$ filled glovebox and their photoresponsivities were measured at specific time under ambient condition.

In the demonstration visible light communication (VLC) system, two open-source electronics platforms (Arduino UNO) were used as drivers to convert digital data to high/low voltage level or vice versa. The photocurrent signal of the perovskite photodetector was convert to high/low voltage level using a current preamplifier before inputted to the driver. The audio signal was transmitted as analog signal which was modulated as the change of LED light intensity. The analog signal was received by the perovskite photodetector and then amplified by a preamplifier before driving the loudspeaker.

\section{Acknowledgements}

This work is financially supported by the National Natural Science Foundation of China (51472164), the 1000 Talents Program for Young Scientists of China, Shenzhen Peacock Plan (KQTD2016053112042971), the Educational Commission of Guangdong Province (2015KGJHZ006, 2016KCXTD006), the Science and Technology Planning Project of Guangdong Province (2016B050501005), Natural Science Foundation of SZU (000050), a China Postdoctoral Science Foundation (2017M622744, 2018T110886), an ERC Starting Grant (717026), the European Commission Marie Skłodowska-Curie Actions (691210), and the Swedish Government Strategic Research Area in Materials Science on Functional Materials at Linköping University (Faculty Grant SFO-Mat-LiU no. 2009-00971).

\section{References}

[1] S. Donati, Meas. Sci. Technol. 2001, 12, 653.

[2] S. F. Tedde, J. Fu, M. V. Kovalenko, T. Rauch, M. Bo, U. Lemmer, W. Heiss, O. 
Hayden, Nat. Photonics 2009, 3, 332.

[3] J. Clark, G. Lanzani, Nat. Photonics 2010, 4, 438.

[4] F. P. G. D. Arquer, A. Armin, P. Meredith, E. H. Sargent, Nat. Rev. Mater. 2017, 2, 16100.

[5] X. Hu, X. Zhang, L. Liang, J. Bao, S. Li, W. Yang, Y. Xie, Adv. Funct. Mater. 2014, 24, 7373.

[6] Y. Lee, J. Kwon, E. Hwang, C. H. Ra, W. J. Yoo, J. H. Ahn, J. H. Park, J. H. Cho, Adv. Mater. 2015, 27, 41.

[7] H. Deng, X. Yang, D. Dong, B. Li, D. Yang, S. Yuan, K. Qiao, Y. B. Cheng, J. Tang, H. Song, Nano Lett. 2015, 15, 7963.

[8] L. Dou, Y. (Micheal) Yang, J. You, Z. Hong, W.-H. Chang, G. Li, Y. Yang, Nat. Commun. 2014, 5, 5404.

[9] R. Dong, Y. Fang, J. Chae, J. Dai, Z. Xiao, Q. Dong, Adv. Mater. 2015, 1912.

[10] Q. Lin, A. Armin, D. M. Lyons, P. L. Burn, P. Meredith, Adv. Mater. 2015, 27, 2060.

[11] Y. Fang, Q. Dong, Y. Shao, Y. Yuan, J. Huang, Nat. Photonics 2015, 9, 679.

[12] Y. Fang, J. Huang, Adv. Mater. 2015, 7, 2804.

[13] C. Bao, W. Zhu, J. Yang, F. Li, S. Gu, Y. Wang, T. Yu, J. Zhu, Y. Zhou, Z. Zou, ACS Appl. Mater. Interfaces 2016, 8, 23868.

[14] C. Bao, Z. Chen, Y. Fang, H. Wei, Y. Deng, X. Xiao, L. Li, J. Huang, Adv. Mater. 2017, 29, 1703209.

[15] Y. Guo, C. Liu, H. Tanaka, E. Nakamura, J. Phys. Chem. Lett. 2015, 6, 535.

[16] K. C. Kwon, K. Hong, Q. V. Le, S. Y. Lee, J. Choi, K.-B. Kim, S. Y. Kim, H. W. Jang, Adv. Funct. Mater. 2016, 26, 4213.

[17] W. Deng, X. Zhang, L. Huang, X. Xu, L. Wang, J. Wang, Q. Shang, S.-T. Lee, J. Jie, Adv. Mater. 2016, 28, 2201.

[18] H. Wang, D. H. Kim, Chem. Soc. Rev. 2017, 46, 5204. 
[19] W. Deng, L. Huang, X. Xu, X. Zhang, X. Jin, S.-T. Lee, J. Jie, Nano Lett. 2017, 17, 2482.

[20] K. Domanski, W. Tress, T. Moehl, M. Saliba, M. K. Nazeeruddin, M. Grätzel, Adv. Funct. Mater. 2015, 25, 6936.

[21] L. Shen, Y. Fang, D. Wang, Y. Bai, Y. Deng, M. Wang, Y. Lu, J. Huang, Adv. Mater. 2016, 28, 10794.

[22] L. Shen, Y. Lin, C. Bao, Y. Bai, Y. Deng, M. Wang, T. Li, Y. Lu, A. Gruverman, W. Li, J. Huang, Mater. Horiz. 2017, 4, 242.

[23] Z. Yang, Y. Deng, X. Zhang, S. Wang, H. Chen, S. Yang, J. Khurgin, N. X. Fang, X. Zhang, R. Ma, Adv. Mater. 2018, 30, 1704333.

[24] X. Li, D. Yu, J. Chen, Y. Wang, F. Cao, Y. Wei, Y. Wu, L. Wang, Y. Zhu, Z. Sun, J. Ji, Y. Shen, H. Sun, H. Zeng, ACS Nano 2017, 11, 2015.

[25] M. Shoaib, X. Wang, X. Zhang, X. Wang, H. Zhou, T. Xu, X. Hu, H. Liu, X. Fan, W. Zheng, T. Yang, S. Yang, Q. Zhang, X. Zhu, L. Sun, A. Pan, J. Am. Chem. Soc. 2017, 139, 15592.

[26] J. Song, L. Xu, J. Li, J. Xue, Y. Dong, X. Li, H. Zeng, Adv. Mater. 2016, 28, 4861.

[27] J. Liang, C. Wang, Y. Wang, Z. Xu, Z. Lu, Y. Ma, H. Zhu, Y. Hu, C. Xiao, X. Yi, G. Zhu, H. Lv, L. Ma, T. Chen, Z. Tie, Z. Jin, J. Liu, J. Am. Chem. Soc. 2016, 138, 15829.

[28] J. Liang, P. Zhao, C. Wang, Y. Wang, Y. Hu, G. Zhu, L. Ma, J. Liu, Z. Jin, J. Am. Chem. Soc. 2017, 139, 14009.

[29] M. Kulbak, S. Gupta, N. Kedem, I. Levine, T. Bendikov, G. Hodes, D. Cahen, J. Phys. Chem. Lett. 2016, 7, 167.

[30] X. Li, D. Yu, F. Cao, Y. Gu, Y. Wei, Y. Wu, J. Song, H. Zeng, Adv. Funct. Mater. 2016, 26, 5903.

[31] Y. Li, Z.-F. Shi, S. Li, L.-Z. Lei, H.-F. Ji, D. Wu, T.-T. Xu, Y.-T. Tian, X.-J. Li, J. Mater. Chem. C 2017, 5, 8355. 
[32] X. Liu, D. Yu, F. Cao, X. Li, J. Ji, J. Chen, X. Song, H. Zeng, Small 2017, 13, 1.

[33] B. Yang, F. Zhang, J. Chen, S. Yang, X. Xia, T. Pullerits, W. Deng, K. Han, Adv. Mater. 2017, 29, 1.

[34] J.-H. Cha, J. H. Han, W. Yin, C. Park, Y. Park, T. K. Ahn, J. H. Cho, D.-Y. Jung, J. Phys. Chem. Lett. 2017, 8, 565.

[35] J. Song, Q. Cui, J. Li, J. Xu, Y. Wang, L. Xu, J. Xue, Y. Dong, T. Tian, H. Sun, H. Zeng, Adv. Opt. Mater. 2017, 5, 1.

[36] J. Yang, T. Yu, K. Zhu, Q. Xu, J. Phys. D. Appl. Phys. 2017, 50, 495102.

[37] D. Bryant, N. Aristidou, S. Pont, I. Sanchez-Molina, T. Chotchunangatchaval, S. Wheeler, J. R. Durrant, S. A. Haque, Energy Environ. Sci. 2016, 9, 1655.

[38] Y. Yuan, J. Huang, Acc. Chem. Res. 2016, 49, 286.

[39] T. A. Berhe, W.-N. Su, C.-H. Chen, C.-J. Pan, J.-H. Cheng, H.-M. Chen, M.-C. Tsai, L.Y. Chen, A. A. Dubale, B.-J. Hwang, Energy Environ. Sci. 2016, 9, 323.

[40] S. Bai, Z. Wu, X. Wu, Y. Jin, N. Zhao, Z. Chen, Q. Mei, X. Wang, Z. Ye, T. Song, R. Liu, S. tong Lee, B. Sun, Nano Res. 2014, 7, 1749.

[41] B. Conings, J. Drijkoningen, N. Gauquelin, A. Babayigit, J. D’Haen, L. D’Olieslaeger, A. Ethirajan, J. Verbeeck, J. Manca, E. Mosconi, F. D. Angelis, H. G. Boyen, Adv. Energy Mater. 2015, 5, 1500477.

[42] Y. Kato, L. K. Ono, M. V. Lee, S. Wang, S. R. Raga, Y. Qi, Adv. Mater. Interfaces 2015, 2, 1500195 .

[43] A. Jovicic, J. Li, T. Richardson, IEEE Commun. Mag. 2013, 51, 26.

[44] P. H. Pathak, X. Feng, P. Hu, P. Mohapatra, IEEE Commun. Surv. Tutorials 2015, 17, 2047. 


\section{Supporting Information}

High performance and stable all-inorganic metal halide perovskite-based photodetectors for optical communication application

Chunxiong Bao, Jie Yang, Sai Bai, Weidong Xu, Zhibo Yan, Qingyu Xu, Junming Liu, Wenjing Zhang*, Feng Gao*
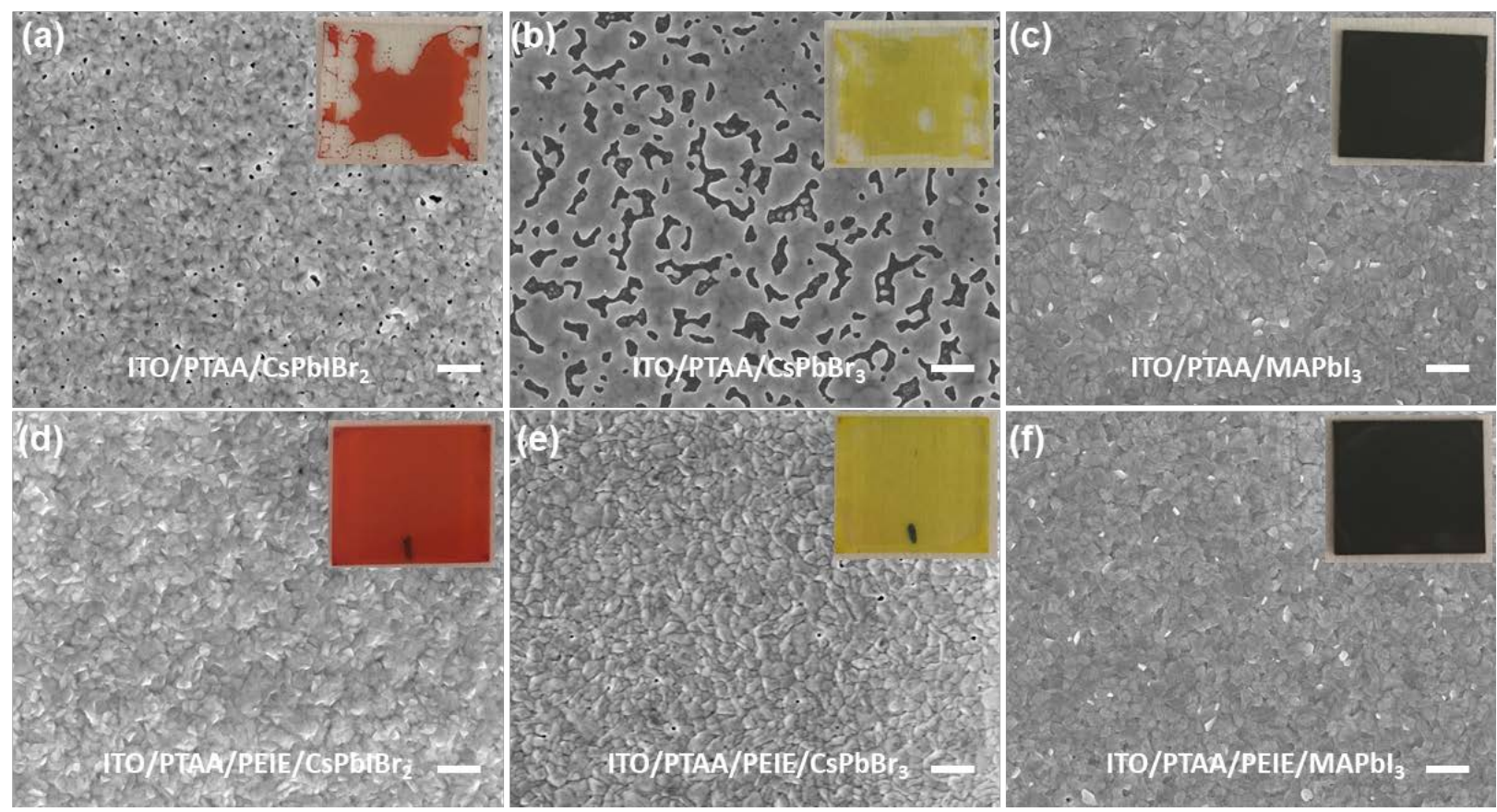

Figure S1. a) to c) Scanning electron microscope (SEM) images of $\mathrm{CsPbIr}_{2}, \mathrm{CsPbBr}_{3}$ and

$\mathrm{CH}_{3} \mathrm{NH}_{3} \mathrm{PbI}_{3}\left(\mathrm{MAPbI}_{3}\right)$ films on ITO/PTAA substrates. d) to f) SEM images of $\mathrm{CsPbIBr}$, $\mathrm{CsPbBr}_{3}$ and $\mathrm{MAPbI}_{3}$ films on ITO/PTAA/PEIE substrates. The inset images are the digital photographs of each films. The scale bars for all images are $1 \mu \mathrm{m}$. 

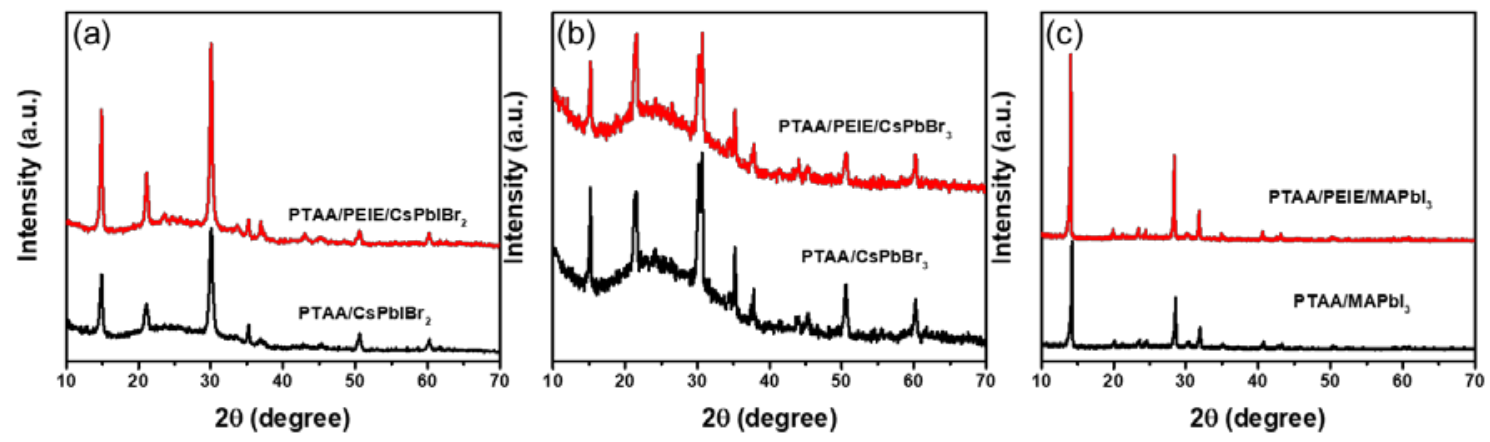

Figure S2. X-ray diffraction spectra of $\mathrm{CsPbIBr}_{2}(\mathrm{a}), \mathrm{CsPbBr}_{3}$ (b) and $\mathrm{CH}_{3} \mathrm{NH}_{3} \mathrm{PbI}_{3}\left(\mathrm{MAPbI}_{3}\right)$

(c) films on ITO/PTAA and ITO/PTAA/PEIE substrates.
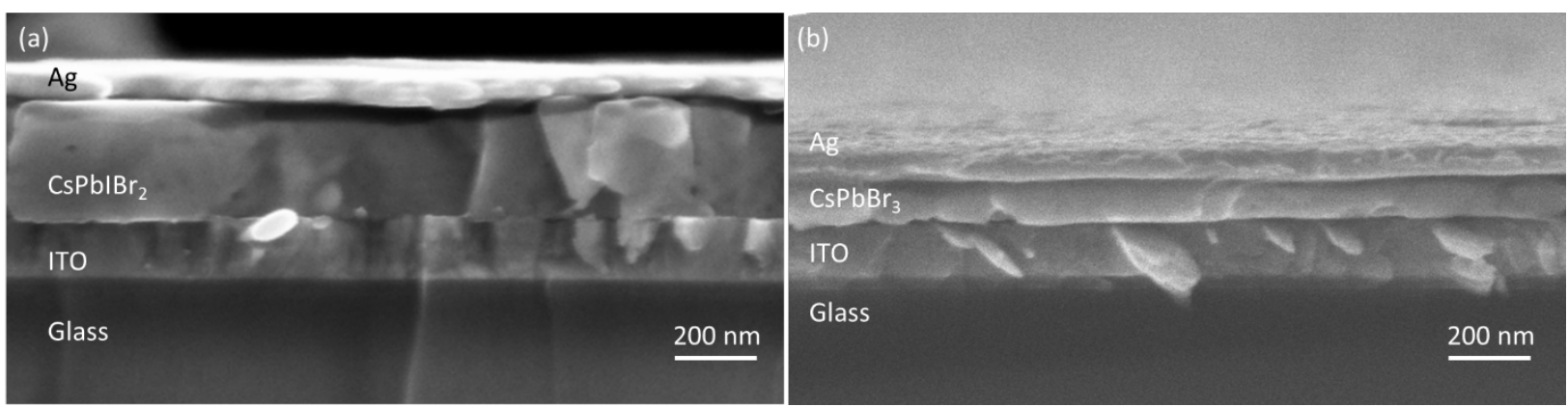

Figure S3. Cross-sectional SEM images of $\mathrm{CsPbIBr}_{2}$ (a) and $\mathrm{CsPbBr}_{3}$ (b) based photodetectors.

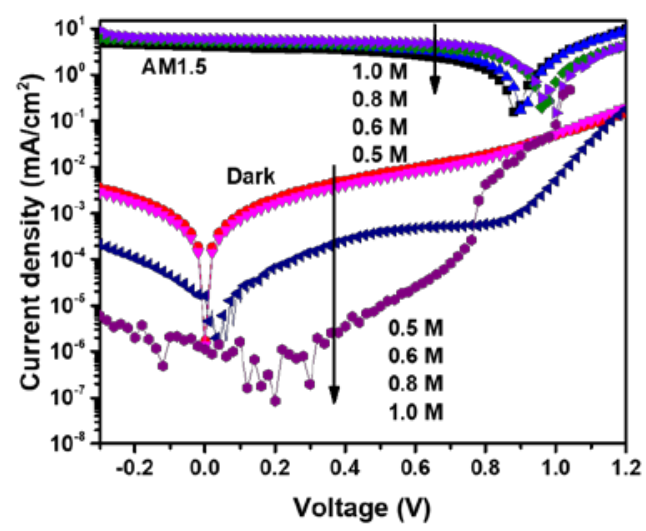

Figure S4. Current density-voltage (J-V) curve of $\mathrm{CsPbIBr}_{2}$ devices whith different perovskite layer thicknesses under dark and illumination with AM1.5 sunsimulator light. 

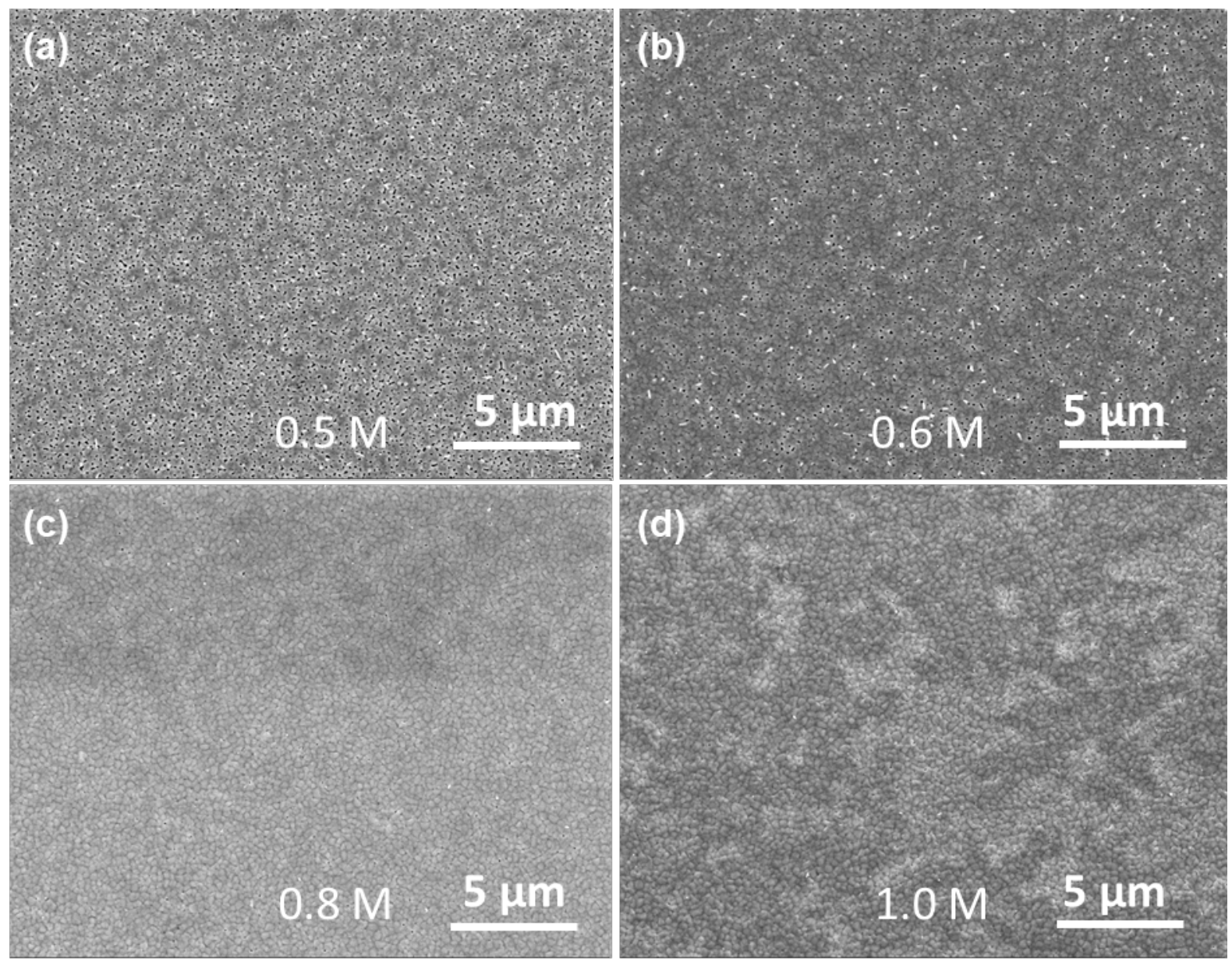

Figure S5. SEM images of $\mathrm{CsPbIBr}_{2}$ fabricated from precursor solutions with concentrations of $0.5 \mathrm{M}(\mathrm{a}), 0.6 \mathrm{M}(\mathrm{b}), 0.8 \mathrm{M}$ (c) and $1.0 \mathrm{M}$ (d).

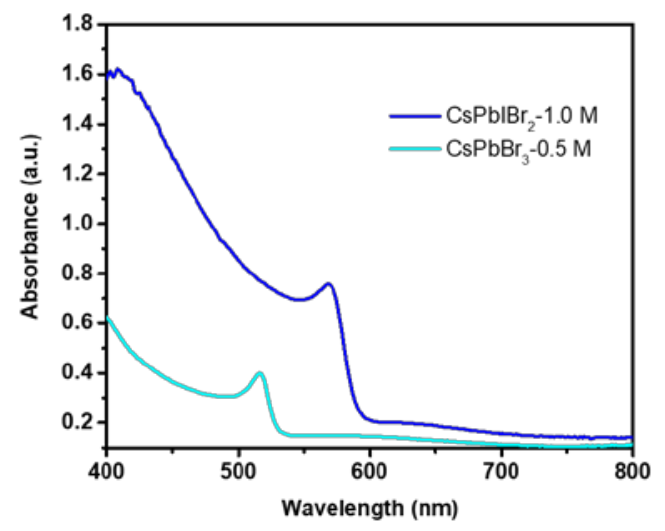

Figure S6. UV-Vis absorption spectra of $\mathrm{CsPbBr}_{3}$ and $\mathrm{CsPbIBr}_{3}$ films. 
$\mathrm{CsPblBr}_{2}$

full area
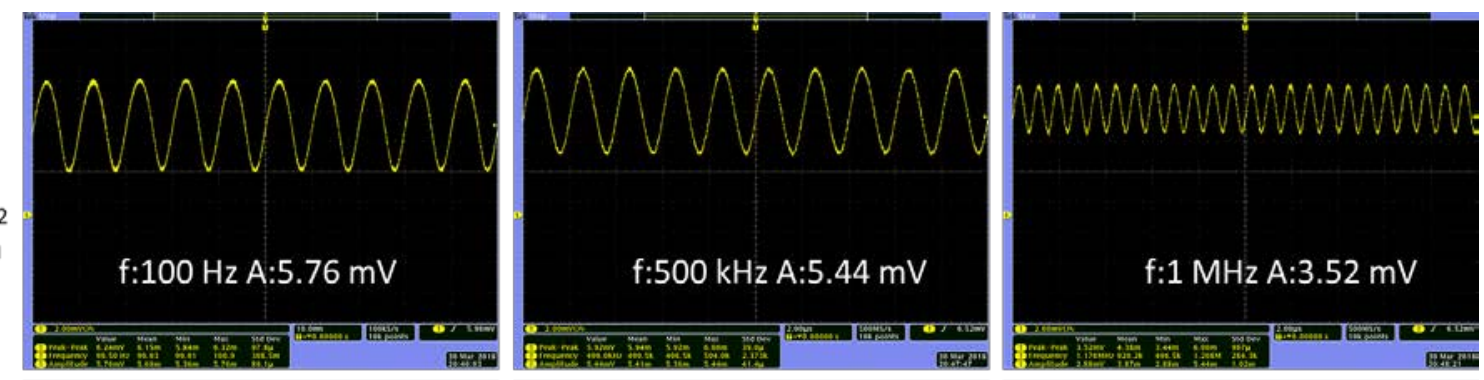

$\mathrm{CsPbBr}_{3}$ full area
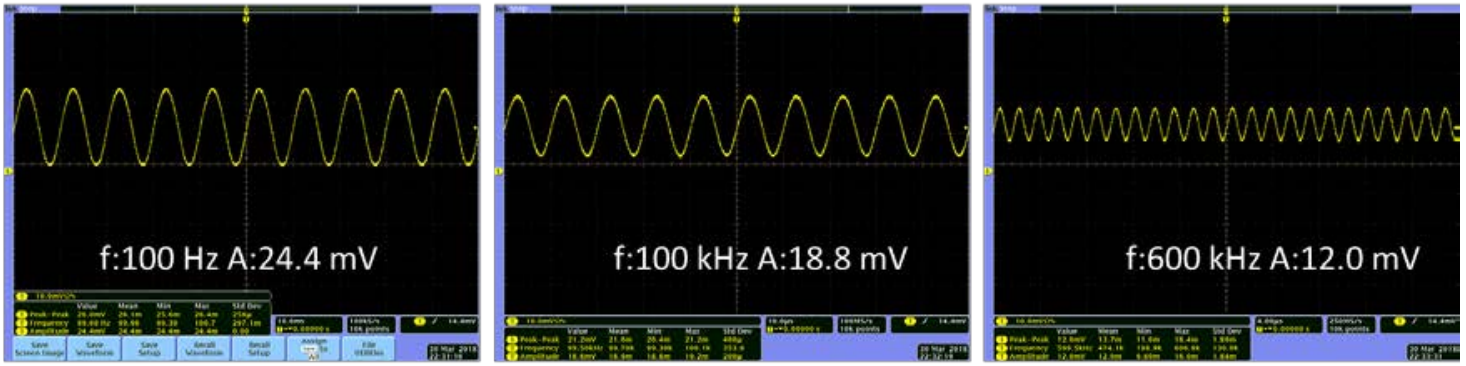

$\mathrm{CsPbIBr}_{2}$ half area
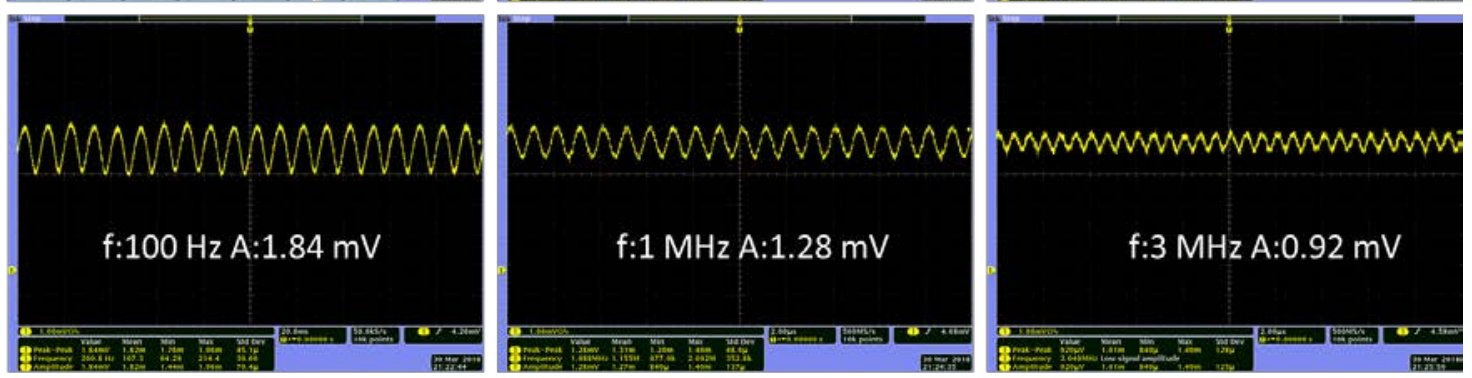

$\mathrm{CsPbBr}_{3}$ half area
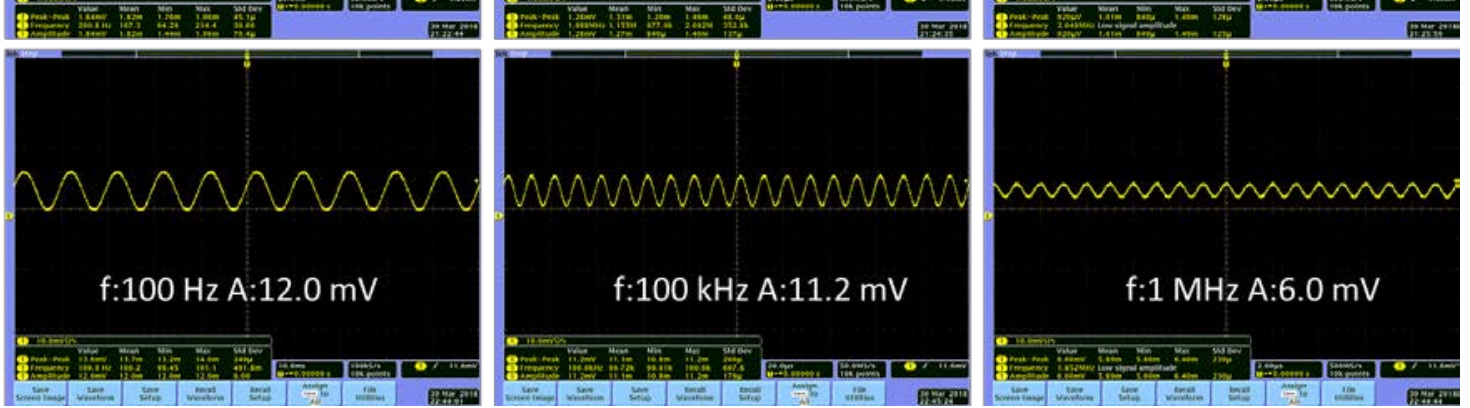

Figure S7. The response waveforms of $\mathrm{CsPbIBr}_{2}$ and $\mathrm{CsPbBr}_{3}$ devices to a sine wave modulated LED light at different frequencies. 

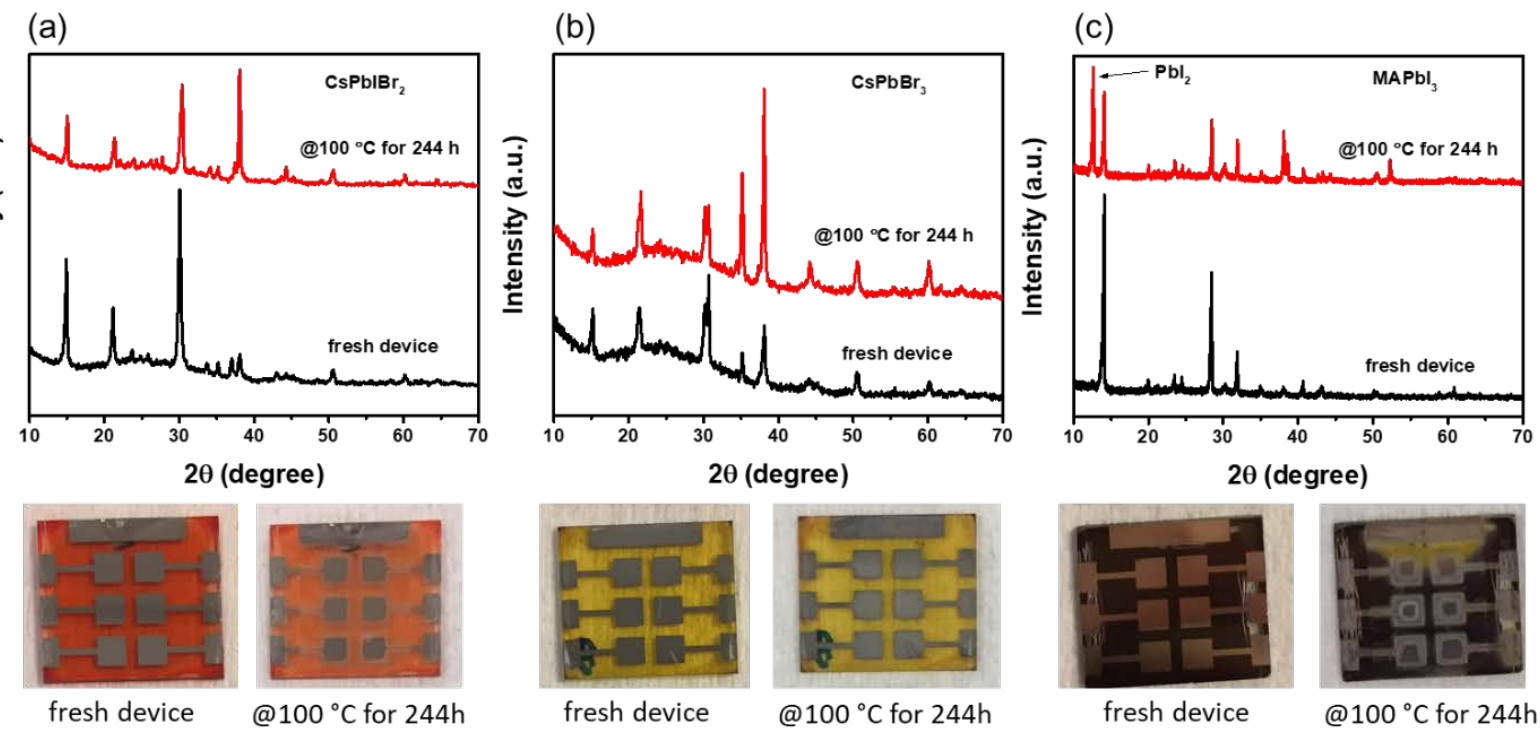

fresh device

@ $100{ }^{\circ} \mathrm{C}$ for $244 \mathrm{~h}$

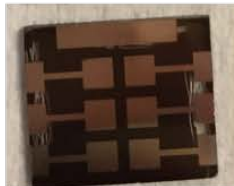

fresh device

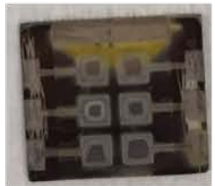

@ $100{ }^{\circ} \mathrm{C}$ for $244 \mathrm{~h}$

Figure S8. X-ray diffraction (XRD) spectra and digital photographs of $\mathrm{CsPbIBr}_{2}(\mathrm{a}), \mathrm{CsPbBr}_{3}$ (b) and $\mathrm{CH}_{3} \mathrm{NH}_{3} \mathrm{PbI}_{3}\left(\mathrm{MAPbI}_{3}\right)$ (c) devices before and after heated at $100{ }^{\circ} \mathrm{C}$ in $\mathrm{N}_{2}$ filled glove box for 244 hours. The obvious $\mathrm{PbI}_{2}$ peak in XRD spectrum of $\mathrm{MAPbI}_{3}$ devices after heated indicates the decomposition of $\mathrm{MAPbI}_{3}$.

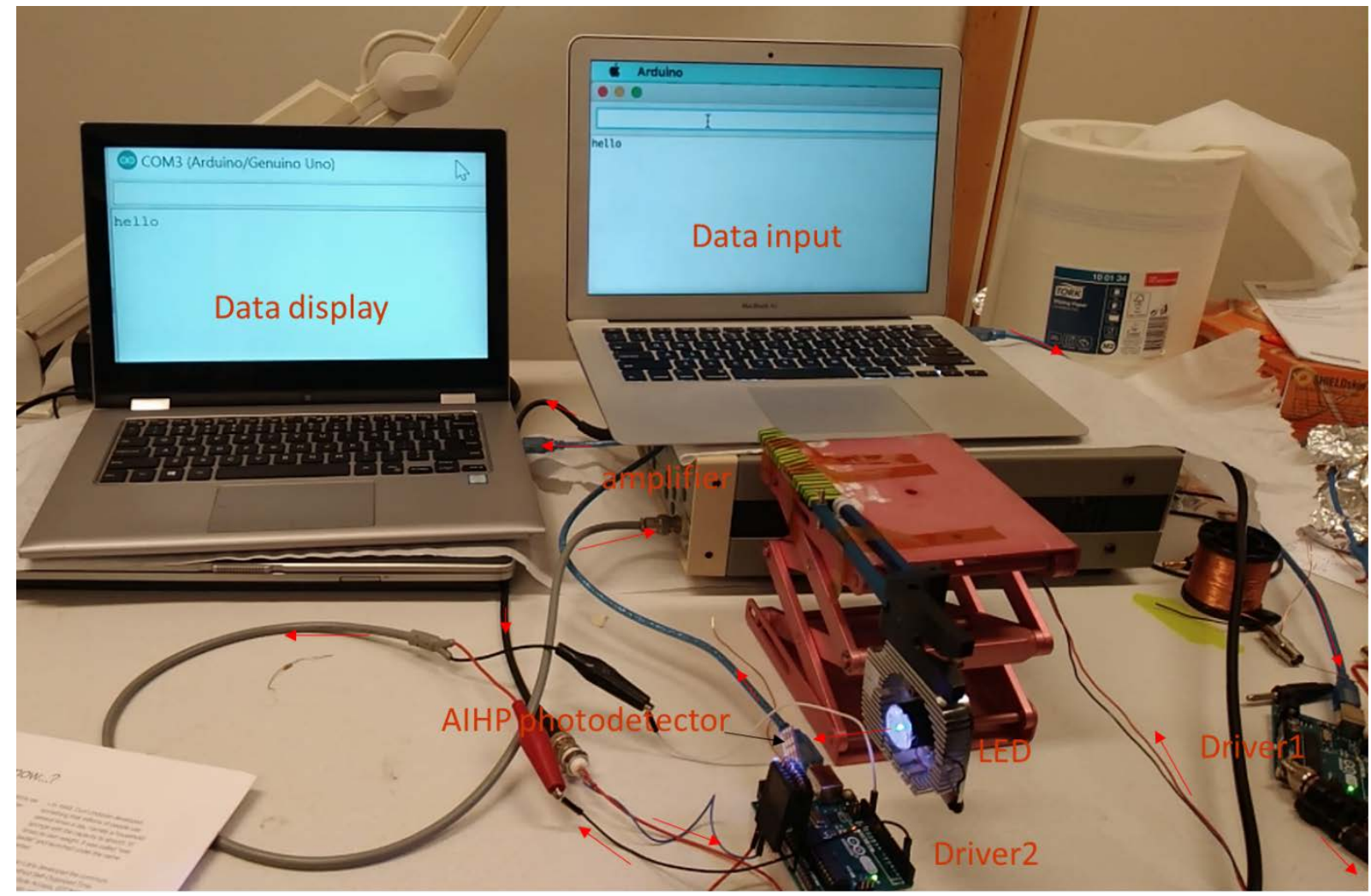


Figure S9. digital photograph of the visible light communication (VLC) system with our allinorganic halide perovskite (AIHP) photodetector as light signal receiver. The red arrows indicate the data flow direction. 